\title{
Toward the Optimization of a PMSG Wind Energy Conversion System On-Grid by a Robust Mixed Controller
}

\author{
Zakariae JAI ANDALOUSSI ${ }^{1 *}$, Abdelhadi RAIHANI ${ }^{1}$, Abdelmounime ELMAGRI ${ }^{1}$, Rachid LAJOUAD ${ }^{1}$
}

${ }^{1}$ Signal Distributed Systems and Artificial Intelligence Laboratory (SSDIA) of the Normal Superior School of Technical Education of Mohammedia ENSETM, BP 159 Mohammedia Principale, Morocco

\begin{abstract}
The grid interconnected Wind Energy Conversion System (WECS) remains a major challenge. To deal with the effect of the intermittent nature of wind speed. This paper presents the design of a combined nonlinear controller based on a sliding mode controller (MSMC) for a wind system. This control technique includes a smooth switching sliding mode observer (SS-SMO) and a non-singular terminal sliding mode controller (NT-SMC). The SS-SMO is used to observe the torque/speed disturbances, while the NT-SMC is used as a regulator. A voltage control technique is adopted to maintain the voltage at the common DC-link. To control the electrical power injected into the grid a Backstepping controller is proposed. The WECS is built around a wind turbine coupled to a Permanent Magnet Synchronous Generator (PMSG). The proposed technique is robust against model uncertainties and external disturbances. In addition, the complexity of the system is reduced by replacing the mechanical speed and position sensors with the estimated parameter. The simulations results show the performances in terms of monitoring of set point, stability, and robustness with respect to the variation of wind speed.
\end{abstract}

\section{Introduction}

The wind generator proposed in this study is a permanent magnet synchronous generator due to its advantages in terms of variable speed and low-frequency converter back-to-back converter. Due to the many advantages cited in the literature [1, 2, 3, 4]. PMSGs have proven to be an appropriate option among the generators available for Wind Turbine Conversion System (WTCS) because they eliminate the need for an additional gearbox and slip ration $[3,4]$. WECS works effectively when used with a fully controlled source side converter (SSC), embedded with appropriate control logic. This configuration facilitates the extraction of optimum wind energy within a certain wind speed range by regulating the speed of the shaft. The high gain classic PI controller [6] improves system dynamics but does not improve the steady-state performance. However, a low controller gain reduces the dynamic response of the system. Various adaptive, intelligent, and robust techniques have been reported for WEGS based on PMSG compared to the conventional PI controller. Robust techniques prove to be the appropriate choice for the rejection of model uncertainties and external disturbances. The SMC technique is gaining increasing interest among robust techniques. For SMC, the main requirements are slip surface selection, law enforcement, and slip mode gains. In addition, the gain in sliding mode depends on the upper limit of the localized disturbances. Due to the use of high gain and discontinuous signum function, SMC encounters a gossip problem [7, 8].

Various methods have been suggested to address the persistent problem in conventional SMC, which includes taking into account different range laws, sliding surfaces, disturbance observer loops, and the introduction of continuous switching functions. Very few contributions are reported on the development of WEGS using the Disturbance Observer technique [9]. The proposed work focuses on maximizing wind energy extraction by controlling the speed of the PMSG tree using the proposed control. Due to the intermittent wind speed, WEGS experiences external disturbances and model uncertainties. The main objective of the designed control is to treat these unknown disturbances. These are introduced because of the unpredictable wind speed and reduce the problem of interference in the system, which is the major drawback of conventional SMC systems.

In order to meet the above objectives, it is proposed to design and implement a robust Sliding Mode Control system. The wind conversion system consists of two threephase full-bridge converters connected back-to-back, socalled rectifier 'Source Side Converter' and inverter 'Grid Side Converter'. The well-known FOC is used to generate the switching pulses for the SSC [10]. The SMC is used to track the maximum power of the wind turbine by regulating the PMSG speed with the possibility of disturbance compensation. It includes SS-SMO with fast and finite convergence. SS-SMO observes the localized disturbance in a distinct loop. It is included in the controller as a feed-forward term that assists in disturbance rejection. Here, the conventional discontinuous sign function is replaced by a smooth hyperbolic tangent function providing smooth switching. In addition, the disturbance compensation loop allows for a small slip gain selection in the speed controller loop, which improves system performance. Given the dynamics of the rotor speed PMSG. NT-SMC is used to control the speed. The shaft speed and rotor position are estimated using the electromotive force counter (EMC) technique, eliminating the need for mechanical speed and position sensors [10, 12, 13].

The GSC is operated using a power control technique because when the WEC system is integrated into the electrical network, the reactive power flow sent to the network must be managed, for this, we propose a new nonlinear algorithm based on the adaptative Backstepping controller. 


\section{Presentation of the studied system}

The basic configuration of the entire system is shown in Figure 1. The system studied consists of three finned rotors with a corresponding mechanical gearbox, a PMSG, two power converters (SSC and GSC), a DC bus voltage and a network filter. The SSC works as a rectifier and the GSC works as an inverter.

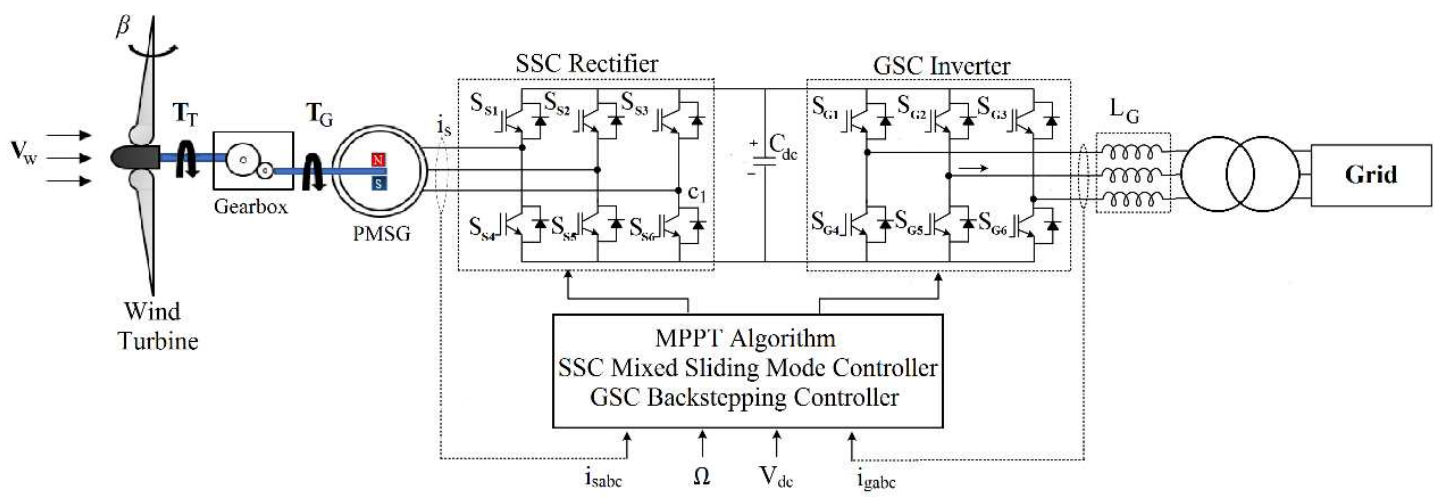

Figure 1. Grid-on Wind Conversion Energy System

\section{Modelling of Wind system components}

\subsection{Wind turbine modelling}

Wind turbines produce electricity using wind power to drive an electric generator. The wind passes over the blades, exerting a rotating force. The rotating blades rotate a shaft inside the nacelle, which enters a gearbox. The gearbox increases the rotational speed at which it is suitable for the generator, which uses magnetic fields to convert rotational energy into electrical energy. The rotor is the component designed to extract energy from the wind. The power extracted from the wind can be defined by the difference between the power in the moving air force and after the rotor. According to the Betz theory [3, 14], the mechanical turbine power $P_{T}$ extracted from wind energy can be written as:

$$
P_{T}=\frac{1}{2} \cdot C_{p}(\lambda, \beta) \cdot \rho \cdot S \cdot V_{w}^{3}=T_{T} \cdot \Omega_{t}
$$

With $C_{p}$ is the wind power coefficient, $\rho$ is the air density $(1,25 \mathrm{~kg} / \mathrm{m}), S$ is the surface and $V_{w}$ is the wind speed $(\mathrm{m} / \mathrm{s})$.

The wind power coefficient $C_{p}$ defines the ability of a wind turbine to capture wind energy through the extracted power ratio to wind power. Function of both pitch angle $\beta$ and tip speed ratio $\lambda$. For fixed pitch $\beta=0^{\circ}$ the only factor affecting the power coefficient is the tip speed ratio $\lambda$, which is given by:

$$
\lambda=\Omega \cdot R / V_{w}
$$

Where, $\Omega$ is the rotor angular velocity $(\mathrm{rad} / \mathrm{s}) . R$ is the wind blade radius $(\mathrm{m})$.

Several models of the power coefficient were introduced in the literature; generally using for particular turbine [15] each turbine has a specific behaviour. It has the advantage of being in the form of a single equation, valid whatever the pitch angle $\beta$ and the velocity ratio $\lambda$. The coefficient of the power $C_{p}$ has for expression:

$$
\begin{array}{ll}
C_{p}(\lambda, \beta)=0,5109 \cdot\left(\frac{116}{\lambda_{i}}-0,4 \cdot \beta-5\right) \cdot e^{-\frac{21}{\lambda_{i}}}+0,0068 \cdot \lambda \\
\text { With: } \quad \lambda_{i}=\frac{1}{\lambda+0,08 \cdot \beta}-\frac{0,035}{\beta^{3}+1}
\end{array}
$$

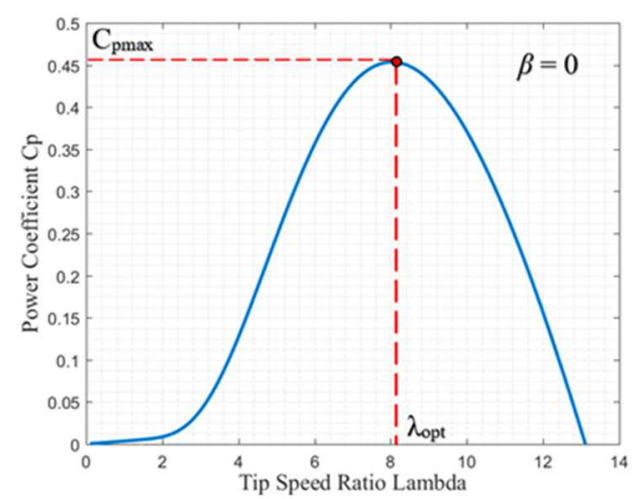

Figure 2. Wind turbine power coefficient versus tip speed ratio for a fixed-pitched

When the pitch angle $\beta=0^{\circ}$, it corresponds to the $C_{p}$ versus $\lambda$ characteristic used in the turbine emulation of this study. It can be seen that a maximum power coefficient $C_{\text {pmax }}=0.48$ is achieved at only one optimum tip speed ration, $\lambda_{\text {opt }}=8.1$.

The turbine torque is obtained by using the equation (1). The expression of the aerodynamic torque $T_{T}$ is then given by:

$T_{T}=\frac{P_{T}}{\Omega_{t}}=\frac{1}{2} \cdot C_{p}(\lambda, \beta) \cdot \rho \cdot S \cdot V_{w}^{3} / \Omega_{t}$

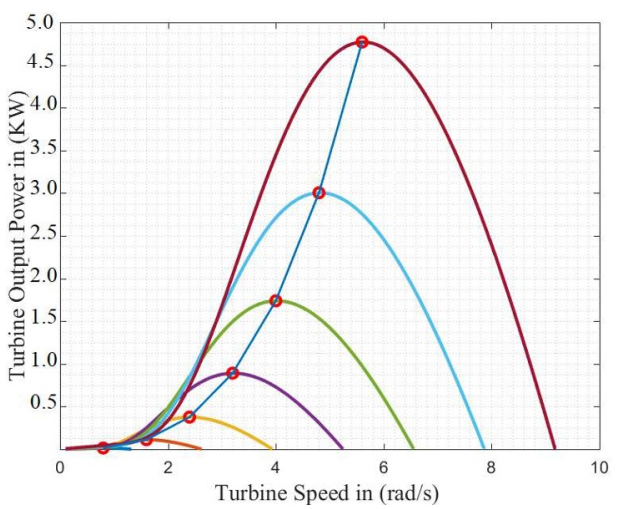

Figure 3. Turbine power-speed characteristics in per unit

The speed and torque of the wind turbine and the PMSG are related respectively by:

$$
T_{T}=G \times T_{G}
$$


The dynamics of the mechanical speed $\Omega$ of the PMSG is obtained by applying the Fundamental Principle of the Dynamics of Rotation FPD:

$$
J \cdot \frac{d \Omega}{d t}=T_{G}-F . \Omega-T_{e m}
$$

Where: $T_{e m}$ is the electromagnetic torque, $J$ is the total inertia, and $F$ is the friction coefficient.

\subsection{Wind turbine Maximum power extraction}

Most wind turbines are constrained to work at constant speeds despite wind speed random variation. In such an operation, achieving maximum wind energy extraction in presence of varying wind speed conditions needs a varying turbine speed operation mode. Specifically, the turbine rotor velocity must be controlled so that its power-speed working point is constantly maintained near the optimal position. This control objective is commonly referred to as Maximum Power Point Tracking (MPPT). In WECS the use of an MPPT controller is essential in the sense of maximum wind energy extraction [16].

There are several techniques to reach the MPP. That they are distinguished into two types: Direct methods, those that do not require prior knowledge of WT characteristics; on the opposite indirect methods such as Optimal Torque Control (OTC) which are used in this article [17]. The principle of OTC is to adjust the PMSG torque to its reference for different wind speeds. It requires the knowledge of the optimal turbine characteristics of the power coefficient $C_{\text {pmax }}$ and tip speed ratio $\lambda_{\text {opt }}$. Using equation (2), the wind speed for the optimal parameters is expressed:

$$
V_{w}=\Omega_{o p t} \cdot R / \lambda_{o p t}
$$

Replacing the wind speed in the expression of the turbine torque calculate the optimal torque:

$$
T_{\text {Tref }}=C_{\text {pmax }} \cdot \rho \cdot \pi \cdot R^{2} \cdot V_{w}^{3} / 2 \cdot \Omega_{o p t}=K_{o p t} \cdot \Omega_{o p t}^{2}
$$

With $K_{o p t}$ is a constant depend on the optimal parameters of the WT. It's defined by:

$$
K_{\text {opt }}=C_{\text {pmax }} \cdot \rho \cdot \pi \cdot R^{5} / 2 \cdot \lambda_{o p t}^{3}
$$

The MPPT controller based on the OTC method is described in the block diagram bellow.

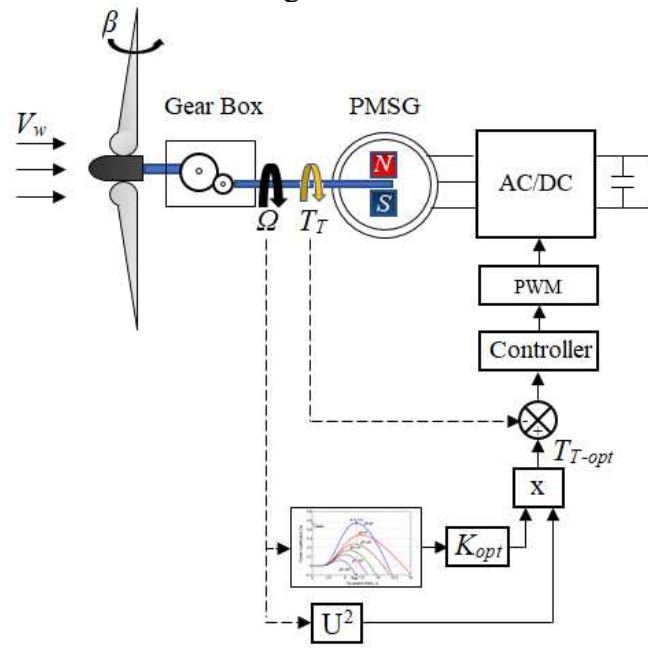

Figure 4. MPPT Block diagram of wind turbine

\subsection{PMSG modelling}

The PMSG model is implemented entirely in $d-q$ coordinates. It means that there are no AC-states in the model. The generator is modelled with DC voltages and currents in a rotor-fixed rotating coordinate system. The equations for the d-axis and q-axis currents are defined in as $[18,19]$ :

$$
\left\{\begin{array}{l}
\frac{d i_{s d}}{d t}=\frac{1}{L_{s d}} \cdot v_{s d}-\frac{R_{s}}{L_{s d}} \cdot i_{s d}+\frac{L_{s q}}{L_{s d}} \cdot \omega_{s} \cdot i_{s q} \\
\frac{d i_{s q}}{d t}=\frac{1}{L_{s q}} \cdot v_{s q}-\frac{R_{s}}{L_{s q}} \cdot i_{s q}-\left(\frac{L_{s d}}{L_{s q}} i_{s d}+\frac{1}{L_{s q}} \cdot \Psi_{p}\right) \cdot \omega_{s}
\end{array}\right.
$$

Where $\left(v_{s d}, v_{s q}\right)$ and $\left(i_{s d}, i_{s q}\right)$ denote the averaged components of the stator voltage and current in $d q$ coordinate coordinates (Park transformation of three-phase stator voltage vector) respectively; $R_{s}$ and $\left(L_{s d}, L_{s q}\right)$ are the stator resistance and inductance; $\Psi_{p}$ the magnetic flux amplitude induced by the rotor permanent magnets in the stator phase; $p$ number of poles pairs.

The equation of the electromagnetic power and torque in the rotor is:

$$
\left\{\begin{array}{l}
P_{e m}=\frac{3}{2} \cdot\left(v_{s d} \cdot i_{s d}+V_{s q} \cdot i_{s q}\right) \\
T_{e m}=\frac{3}{2} \cdot p \cdot\left[\Psi_{p} \cdot i_{s q}+\left(L_{s d}-L_{s q}\right) \cdot i_{s d} \cdot i_{s q}\right]
\end{array}\right.
$$

\subsection{PMSG Speed Estimation}

The requirement for mechanical sensors to measure rotor speed and position is removed by adopting a technique for estimating rotor speed and position using the counter-electromagnetic method [17]. Instead of measuring the stator voltages, these are estimated from the DC voltage $V_{d c}$ and switch sequences of the SSC as follows:

$$
\left[\begin{array}{l}
V_{s a} \\
V_{s b} \\
V_{s c}
\end{array}\right]=\frac{V_{d c}}{3} \cdot\left[\begin{array}{ccc}
2 & -1 & -1 \\
-1 & 2 & -1 \\
-1 & -1 & 2
\end{array}\right] \cdot\left[\begin{array}{c}
V_{s 1} \\
V_{s 3} \\
V_{s 5}
\end{array}\right]
$$

To estimate the position of the rotor $\left(\theta_{S}^{\text {est }}\right)$ and the speed $\left(\Omega^{e s t}\right)$, the PMSG modelling equations, written in the stationary $\alpha-\beta$ reference system, as follows $[20,21]$ :

$$
\left\{\begin{array}{l}
V_{s \alpha}=R_{s} \cdot i_{s \alpha}+L_{s} \cdot \frac{d i_{s \alpha}}{d t}-K_{e m} \cdot p \cdot \Omega^{e s t} \cdot \sin \left(\theta_{s}^{e s t}\right) \\
V_{s \beta}=R_{s} \cdot i_{s \beta}+L_{s} \cdot \frac{d i_{s \beta}}{d t}+K_{e m} \cdot p \cdot \Omega^{e s t} \cdot \cos \left(\theta_{s}^{e s t}\right)
\end{array}\right.
$$

where, $V_{s \alpha}, V_{s \beta}$ and $i_{s \alpha}, i_{s \beta}$ are the stator voltages and currents in stationary $\alpha-\beta$ reference frame. $K_{e m}$ is the backEMF constant of PMSG.

The synchronous rotation of the flux vectors $\Psi_{s \alpha}$ and $\Psi_{s \beta}$, is achieved at steady state that satisfies the conditions $\frac{d i_{s \alpha}}{d t}=0$ and $\frac{d i_{s \beta}}{d t}=0$. At this point, the calculation angle of the stator flux gives the determination of the rotor flow angle $\theta_{s}^{e s t}$. The $\Psi_{s \alpha}$ and the $\Psi_{s \beta}$ are made as:

$$
\left\{\begin{array}{l}
\Psi_{s \alpha}=\int\left(V_{s \alpha}-R_{s} \cdot i_{s \alpha}\right) \cdot d t \\
\Psi_{s \beta}=\int\left(V_{s \beta}-R_{s} \cdot i_{s \beta}\right) \cdot d t
\end{array}\right.
$$

we set: $\Psi_{p \alpha}=\Psi_{s \alpha}-L_{s} . i_{s \alpha}$ and $\Psi_{p \beta}=\Psi_{s \alpha}-L_{s} . i_{s \beta}$

The estimated $\left(\theta_{s}^{\text {est }}\right)$ and $\left(\Omega^{\text {est }}\right)$ are determined by following the equations: 


$$
\left\{\begin{array}{c}
\theta_{s}^{e s t}=\tan ^{-1}\left(\Psi_{p \beta} / \Psi_{p \alpha}\right) \\
\Omega^{\text {est }}=d \theta_{s}^{\text {est }} / d t
\end{array}\right.
$$

\subsection{Design of the Sliding mode observer}

The SS-SMO is used to observe the sudden change in $T_{G}$, considered an unknown disturbance due to the intermittent nature of the wind speed. In order to reduce the adverse effect of the variable $T_{G}$ on the FOC, it is desirable to observe the perturbation and include it in the FOC. Due to the high switching frequency of the controller, the change of $T_{G}$ is dominant, resulting in a slower variation of the control cycle. Thus, the rate of change is assumed negligible during this change. The SS-SMO scheme is shown in Figure 5.

The speed and torque dynamics of the system using (8) and (9), are presented as:

$$
\left\{\begin{array}{l}
\dot{T}_{G}=0 \\
\dot{\Omega}^{e s t}=\frac{1}{J}\left(T_{G}-T_{e m}-T_{f}\right)=\frac{1}{J}\left(T_{G}-K_{e} i_{s q}-K_{f} \Omega^{e s t}\right)
\end{array}\right.
$$

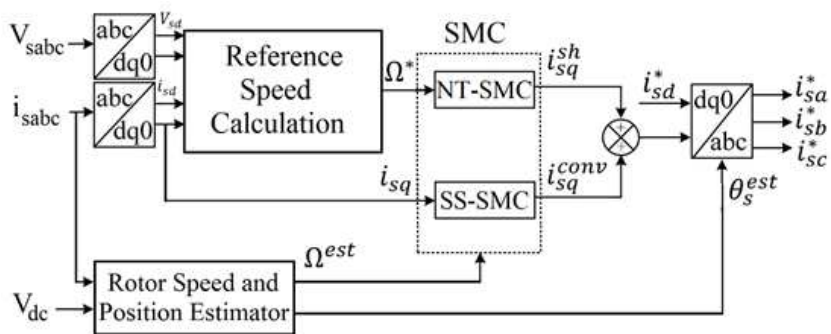

Figure 5. Designed Sliding Mode Controller for SSC

The conventional disturbance observer incorporating the sliding surface is designed with the observation of $\widehat{\Omega}^{e s t}$ and the $\widehat{T}_{G}$ as:

$$
\left\{\begin{array}{l}
\dot{\hat{T}}_{G}=k_{1} \cdot \operatorname{sign}(s) \\
\dot{\widehat{\Omega}}^{e s t}=\frac{\hat{T}_{G}}{J}-\frac{K_{e}}{J} \cdot i_{s q}-\frac{K_{f}}{J} \cdot \widehat{\Omega}^{e s t}+k_{2} \cdot \operatorname{sign}(s)
\end{array}\right.
$$

Where, $k_{1}$ and $k_{2}$ are the feedback gains and the sliding mode of the SMO, respectively.

The sliding surface ( $\mathrm{s}$ ) is defined as the difference between the observed speed $\left(s=\widehat{\Omega}^{e s t}-\Omega^{e s t}\right)$. Because of the nature of the signum switching function $(\operatorname{sign}(s))$ the observer encounters chattering problem.

The mentioned problem is resolved by using a smooth hyperbolic tangent function $(\tanh (s))$. Although, $\tanh (s)$ introduces residual errors, it provides smooth switching. The observer equations become:

$$
\left\{\begin{array}{l}
\dot{\hat{T}}_{G}=k_{1} \cdot \tanh (s) \\
\dot{\widehat{\Omega}}^{e s t}=\frac{\hat{T}_{G}}{J}-\frac{K_{e}}{J} \cdot i_{s q}-\frac{K_{f}}{J} \cdot \widehat{\Omega}^{e s t}+k_{2} \cdot \tanh (s)
\end{array}\right.
$$

The dynamic error of the speed and torque are presented:

$$
\left\{\begin{array}{l}
\dot{\tilde{T}}_{G}=\dot{\hat{T}}_{G}-\dot{T}_{G}=k_{1} \cdot \tanh (s) \\
\dot{\widetilde{\Omega}}^{e s t}=\dot{\widehat{\Omega}}^{e s t}-\dot{\Omega}^{e s t}=\frac{\widehat{T}_{G}}{J}-\frac{K_{f}}{J} \cdot \widetilde{\Omega}^{e s t}+k_{2} \cdot \tanh (s)
\end{array}\right.
$$

To study the stability, we consider the standard function of Lyapunov:

$$
V=\frac{1}{2} \cdot s^{2}
$$

The derivative is given by:

$$
\begin{aligned}
\dot{V}=s \dot{s} & =s\left(\frac{\widehat{T}_{G}}{J}-\frac{K_{f}}{J} \cdot \widetilde{\Omega}^{e s t}+k_{2} \cdot \tanh (s)\right) \\
& =-\frac{K_{f}}{J} \cdot s^{2}+\left(\frac{\hat{T}_{G}}{J}+k_{2} \cdot \tanh (s)\right) \cdot s
\end{aligned}
$$

As $K_{f}$ and $J$ are positive values, the asymptotic stability of the SS-SMO is possible when $\dot{V}<0$. It can be achieved when: $\left(\frac{\hat{T}_{G}}{J}+k_{2} \cdot \tanh (s)\right) . s<0$.

The following conditions are:

$$
\left\{\begin{array}{l}
\frac{\widehat{T}_{G}}{J}-k_{2}>0 \text { if } s<0 \\
\frac{\widehat{T}_{G}}{J}-k_{2} \leq 0 \text { if } s \geq 0
\end{array}\right.
$$

The SS-SMO must be asymptotic stable for the condition on $k_{2}$ where $\left|\frac{\hat{T}_{G}}{J}\right| \geq k_{2}$.

At steady state $s=0$ and $\dot{s}=0$. The dynamics error becomes:

$$
\left\{\begin{array}{l}
\widehat{T}_{G} / J=-k_{1} \tanh (s) \\
\dot{\widetilde{T}}_{G}=k_{2} \tanh (s)
\end{array}\right.
$$

From this equation, we can write:

$$
\dot{\tilde{T}}_{G}=-\frac{k_{2}}{J \cdot k_{1}} \cdot \widehat{T}_{G}
$$

This differential equation admits as solution:

$$
\tilde{T}_{G}=\widetilde{T}_{G}(0) \cdot \exp \left(-\frac{k_{2}}{J \cdot k_{1}}\right)
$$

Proper values for $k_{1}$ and $k_{2}$ are chosen for the SS-SMO to guarantee its stability through Lyapunov analysis. The SS-SMO with the smooth hyperbolic tangent function is shown in Figure 6. From the observed a feed-forward term is calculated as $i_{s q}^{\text {conv }}=k_{3} . \widehat{T}_{G}$.

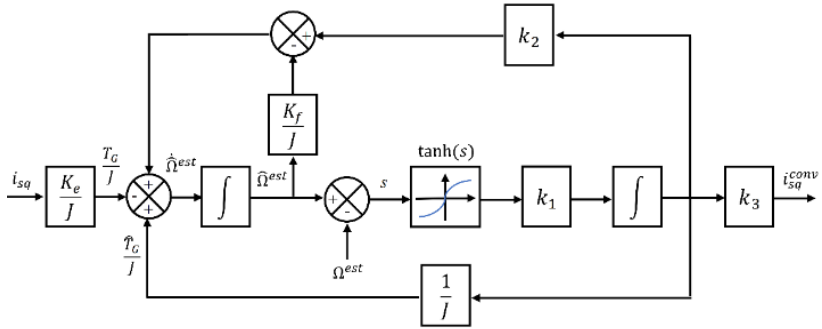

Figure 6. Bloc diagram of the smooth switching SMO

\subsection{Design of Non-Singular Sliding Mode Controller}

The objective of the NT-SMC is to track the desired PMSG speed $\left(\Omega^{e s t}\right)$ that is generated according to the variable wind speed. The NT-SMC is used in the speed control loop of the field oriented controlled PMSG. The speed dynamics of the PMSG is considered to generate the reference $q$-axis current of the speed control loop by replacing in (17) $i_{s q}$ with $i_{s q}^{s h}$ as:

$$
\begin{aligned}
& \dot{\Omega}^{e s t}=\frac{T_{G}}{J}-\frac{K_{e}}{J} i_{s q}^{s h}-\frac{K_{f}}{J} \Omega^{e s t} \\
& \dot{\Omega}^{e s t}=-\frac{K_{e}}{J} i_{s q}-\frac{K_{f}}{J} \Omega_{r e f}^{e s t}+\varepsilon_{t}
\end{aligned}
$$

Where, $\varepsilon_{t}$ is the lumped disturbances due to tracking errors and the turbine torque, which is considered as bounded, defined as:

$$
\varepsilon_{t}=-\frac{K_{e}}{J}\left(i_{s q}^{s h}-i_{s q}\right)+\frac{K_{f}}{J}\left(\Omega_{r e f}^{e s t}-\Omega^{e s t}\right)+\frac{T_{G}}{J}
$$

The state variables are considered as: 


$$
\left\{\begin{array}{l}
x_{1}=\Omega_{r e f}^{e s t}-\Omega^{e s t} \\
\dot{x}_{1}=x_{2}=\dot{\Omega}_{r e f}^{e s t}-\dot{\Omega}^{e s t} \\
\ddot{x}_{2}=\ddot{\Omega}_{r e f}^{e s t}-\ddot{\Omega}^{e s t}
\end{array}\right.
$$

In NT-SMC, a nonlinear term is used to design the nonlinear sliding mode collector defined as:

$$
S_{s}=x_{1}+\frac{1}{\sigma} \cdot x_{2}^{m / n}
$$

With $\sigma, m$ and $n>0$. The sliding mode surface above guarantees the convergence to zero in a finite time interval $\left(t_{s}\right)$. Using the equations (24), (38) and (29), the output of the NT-SMC based speed controller with positive gains is expressed as:

$$
i_{s q}^{s h}=-\frac{J}{K_{s}} \int_{0}^{t}\left(\begin{array}{c}
\ddot{\Omega}_{r e f}^{e s t}+\frac{K_{f}}{J} \dot{\Omega}_{r e f}^{e s t}+\frac{\sigma . n}{m} x_{2}^{\left(2-\frac{m}{n}\right)} \\
+\lambda_{1} \operatorname{sign}\left(S_{s}\right)+\lambda_{2} S_{s}
\end{array}\right) d t
$$

$\lambda_{1}$ and $\lambda_{2}$ are positive constant of the NT-SMC.

To study the stability of the sliding surface, the following Lyapunov function is selected:

$$
V=\frac{1}{2} \cdot S_{S}^{2}
$$

The calculation of the derivative is given by:

$$
\begin{aligned}
\dot{V} & =S_{S} \dot{S}_{S}=S_{S}\left(\dot{x}_{1}+\frac{m}{\sigma \cdot n} x_{2}^{\left(\frac{m}{n}-1\right)} \dot{x}_{2}\right) \\
& =S_{S} \cdot\left(\frac{m}{\sigma \cdot n} \cdot x_{2}^{\left(\frac{m}{n}-1\right)}\right)\left(-\lambda_{1} \cdot \operatorname{sign}\left(S_{S}\right)-\lambda_{2} \cdot S_{S}\right) \\
& =S_{S}\left(\frac{m}{\sigma \cdot n} \cdot x_{2}^{\left(\frac{m}{n}-1\right)}\right)\left(-\lambda_{1} \cdot\left|S_{S}\right|-\lambda_{2} \cdot S_{S}^{2}\right)
\end{aligned}
$$

The sliding surface collector is asymptotically stable assuring its finite time convergence. The SS-SMO observes the variation in $T_{G}$ with variable wind speed and the observed value $i_{s q}^{\text {conv }}$ is added as the feed-forward term with the component of speed control loop $i_{s q}^{s h}$ to limit the influence of the variation on the generator speed. The resultant reference q-axis stator current $i_{s q}^{*}$ of the PMSG defined as [20, 21]:

$$
\begin{gathered}
i_{s q}^{*}=i_{s q}^{s h}+i_{s q}^{c o n v} \\
i_{s q}^{*}=k_{3} T_{G}-\frac{J}{K_{s}} \int_{0}^{t}\left(\begin{array}{c}
\ddot{\Omega}_{r e f}^{e s t}+\frac{K_{f}}{J} \dot{\Omega}_{r e f}^{e s t}+\frac{\sigma . n}{m} x_{2}^{\left(2-\frac{m}{n}\right)} \\
+\lambda_{1} \operatorname{sign}\left(S_{s}\right)+\lambda_{2} S_{s}
\end{array}\right) d t
\end{gathered}
$$

As FOC is used, the $i_{s d}^{*}$ is taken as 0 .

\subsection{Grid side Backstepping Controller}

The grid-side inverter is employed to regulate the DCBus voltage at its reference value and to synchronize the AC power produced by the PMSG with the electrical network. The control strategy is based on the nonlinear Backstepping controller [22]. It is also allowed to control the active and reactive power injected into the grid. Which means the regulation of the power factor during the wind velocity and solar irradiation random changes.

The GSC dynamic model can be described by the following equations:

$$
\left\{\begin{array}{l}
v_{i d}-v_{g d}=R_{f} \cdot i_{d G}+L_{f} \cdot \frac{d i_{d G}}{d t}-L_{f} \cdot \omega \cdot i_{q G} \\
v_{i q}-v_{g q}=R_{f} \cdot i_{q G}+L_{f} \cdot \frac{d i_{q G}}{d t}+L_{f} \cdot \omega \cdot i_{d G}
\end{array}\right.
$$

where $v_{i d}, v_{i q}$ are the inverter voltage on the $d$ - $q$ frame; $v_{g d}, v_{g q}$ are the grid voltage components in the d-axes and $q$-axes; $i_{g d}, i_{g q}$ are the d-axes and q-axes grid current. $R_{f}$, $L_{f}$ are the filter resistance and inductance respectively.

The dynamic DC-link voltage can be extracted from the current expression:

$$
i_{C}=i_{D C}-i_{\text {Inv }}
$$

Developing the equation below, we have the expression of the DC-link voltage.

$$
\begin{gathered}
C \frac{d v_{D C}}{d t}=i_{D C}-\frac{3}{2} \frac{v_{g d}}{v_{D C}} i_{g d} \\
C v_{D C} \frac{d v_{D C}}{d t}=v_{D C} i_{D C}-\frac{3}{2} v_{g d} i_{g d}=P_{D C}-P_{G} \\
\frac{d v_{D C}^{2}}{d t}=\frac{2}{C}\left(P_{D C}-\frac{3}{2} v_{g d} i_{g d}\right)
\end{gathered}
$$

The control of the active and reactive power can be accomplished by controlling $i_{g d}$ and $i_{g q}$. Also, to assure the unity power factor, we fixed the quadrature grid current to zero. The grid direct current is determined by the control of the DC bus.

Step 1: DC-link voltage control, the tracking error is given by:

$$
\left\{\begin{array}{l}
\varepsilon_{D C}=v_{D C}^{2 *}-v_{D C}^{2} \\
\dot{\varepsilon}_{D C}=\dot{v}_{D C}^{2 *}-\frac{1}{C}\left(P_{D C}-\frac{3}{2} \cdot v_{G} \cdot i_{d G}^{*}\right)
\end{array}\right.
$$

Consequently, the grid direct current $i_{g d}$ is used as a virtual variable to control the DC-bus error. The new current reference is defined by:

$$
i_{g d}^{*}=\frac{2}{3} \frac{P_{D C}}{V_{G}}-\frac{C}{3 \cdot V_{G}} \cdot\left(\frac{d v_{D C}^{2 *}}{d t}+k_{D C} \cdot \varepsilon_{D C}\right)
$$

where $k_{D C}$ is the feedback constant of the closed loop. To achieve the regulation of the direct and quadrature grid current.

Step 2: Direct and quadrature grid current errors respectively:

$$
\begin{aligned}
& \left\{\begin{array}{l}
\varepsilon_{d}=i_{d G}^{*}-i_{d G} \\
\dot{\varepsilon}_{d}=i_{d G}^{*}-\frac{1}{L_{f}}\left(e_{d}-v_{G}-R_{f} \cdot i_{d G}+L_{f} \cdot \omega \cdot i_{q G}\right)
\end{array}\right. \\
& \left\{\begin{array}{l}
\varepsilon_{q}=i_{q G}^{*}-i_{q G} \\
\dot{\varepsilon}_{q}=\dot{i}_{q G}^{*}-\frac{1}{L_{F}}\left(e_{q}-R_{F} \cdot i_{q G}-L_{F} \cdot \omega \cdot i_{d G}\right)
\end{array}\right.
\end{aligned}
$$

So, based on the equations (44) and (45), we can rewrite:

$$
\dot{\varepsilon}_{D C-B u s}=-k_{D C-B u s} \cdot \varepsilon_{D c-B u s}+\frac{3}{C} \cdot v_{G} \cdot \varepsilon_{d}
$$

The backstepping control laws will be as follows:

$$
\left\{\begin{array}{c}
V_{d}^{*}=V_{d G}+\frac{3 \cdot L_{f} \cdot V_{d G}}{C} \varepsilon_{D c-B u s}+R_{f} \cdot i_{d G}^{*} \\
+L_{f} \cdot \frac{d i_{d G}^{*}}{d t}-L_{f} \cdot \omega \cdot i_{q G}+L_{f} \cdot k_{d} \cdot \varepsilon_{d} \\
V_{q}^{*}=R_{f} \cdot i_{q G}^{*}+L_{f} \cdot \omega \cdot i_{d G}+L_{f} \cdot k_{q} \cdot \varepsilon_{q}
\end{array}\right.
$$

With $k_{d}, k_{q}$ the design positive constant of the closed loop to ensure the dynamic stability.

\section{Simulation Results and Discussions}

As a perspective to approve, the effectiveness of the proposed sliding mode/Backstepping controllers to the wind conversion system, many simulations were carried 
out using the software Matlab/Simulink under the system parameters listed in table 1.

Table 1. Electrical characteristics of the WT PMSG

\begin{tabular}{|l|c|c|}
\hline Nominal power & $P_{g}$ & $3 \mathrm{~kW}$ \\
\hline Voltage (line-line) & $V_{s}$ & $400 \mathrm{~V}$ \\
\hline Stator resistance & $R_{s}$ & $2.75 \mathrm{~m} \Omega$ \\
\hline Stator inductance & $L_{s}$ & $2.6 \mathrm{mH}$ \\
\hline Pole pairs & $p$ & 2 \\
\hline Moment of inertia & $J$ & 1.4 \\
\hline Friction factor & $F$ & 0.97 \\
\hline Magnet flux & $\Psi_{p}$ & $0.89 \mathrm{~Wb}$ \\
\hline Blade Radius & $R_{B}$ & $3 \mathrm{~m}$ \\
\hline
\end{tabular}

The design parameters are given in the following values that proved to be appropriate:

$$
\begin{gathered}
\lambda_{1}=0.98 ; \lambda_{2}=0.48 ; k_{f}=5.74 ; \\
k_{D C}=150 ; k_{d}=25 ; k_{q}=55
\end{gathered}
$$

The WECS performances are illustrated by Fig.7-14. According to the control design the remaining closed-loop inputs are kept constant namely:

$i_{\text {sdref }}=0 ; v_{\text {DCref }}=400 \mathrm{~V} ; i_{\text {gqref }}=0 \mathrm{Kvar}$

The considered wind speed profile is shown in Figure 7. It is seen that wind velocity varies randomly over a wide range.

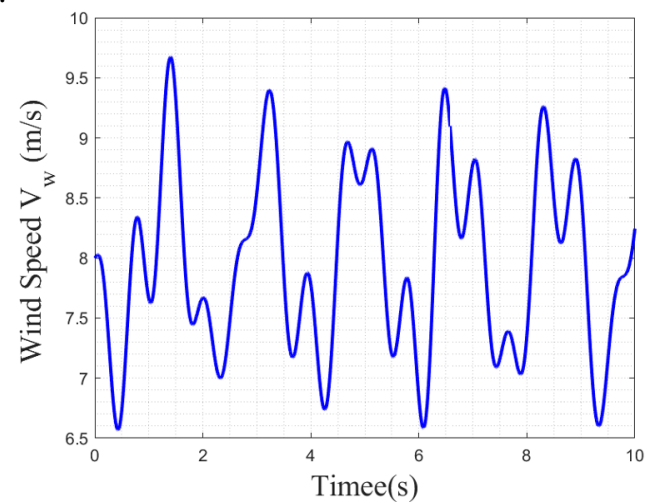

Figure 7. Wind speed profile

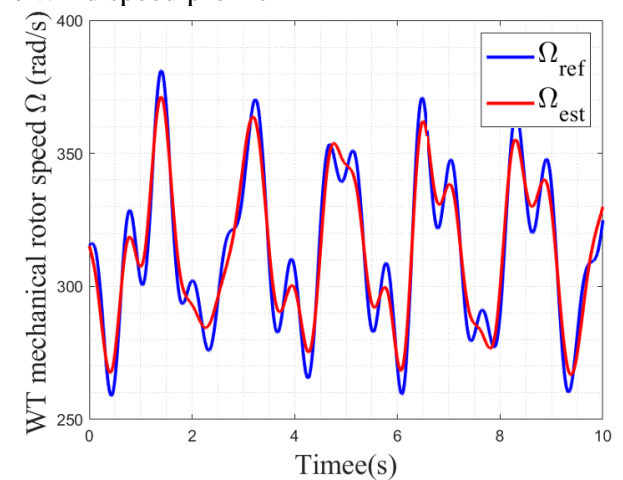

Figure 8. WT mechanical rotor speed estimated and reference

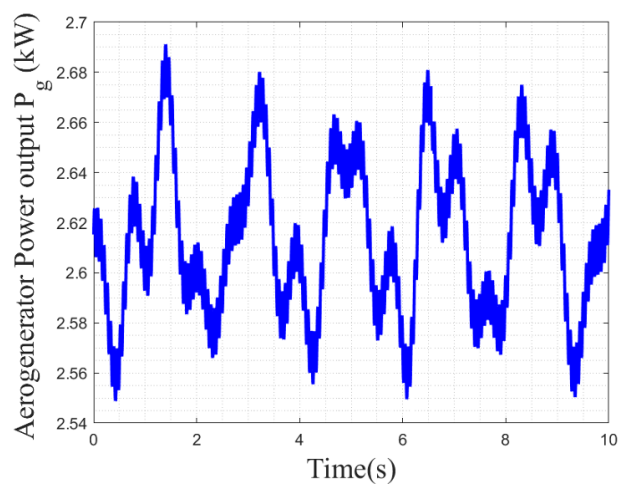

Figure 9. WECS output active power

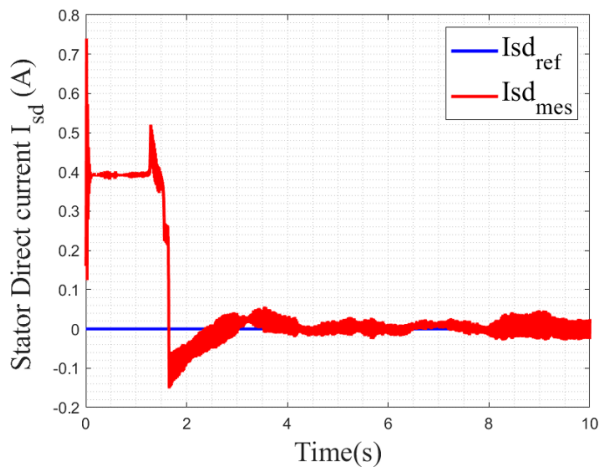

Figure 10. $d$-axis component stator current

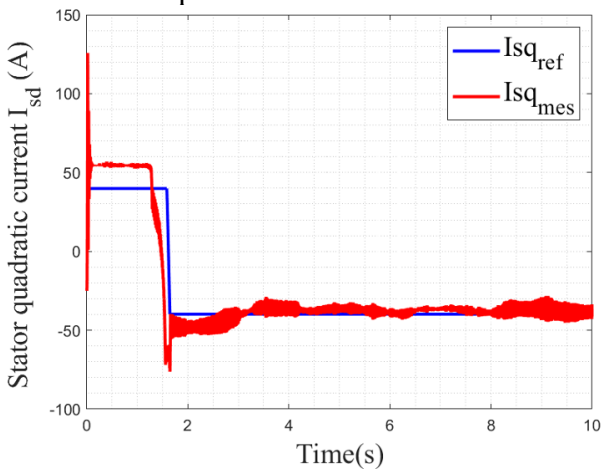

Figure 11. $q$-axis component stator current

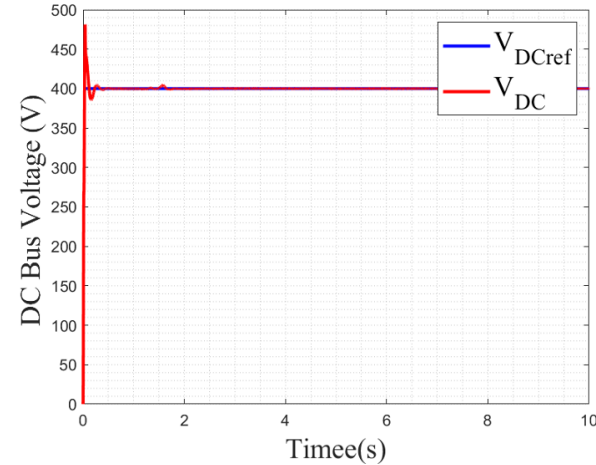

Figure 12. DC-link voltage

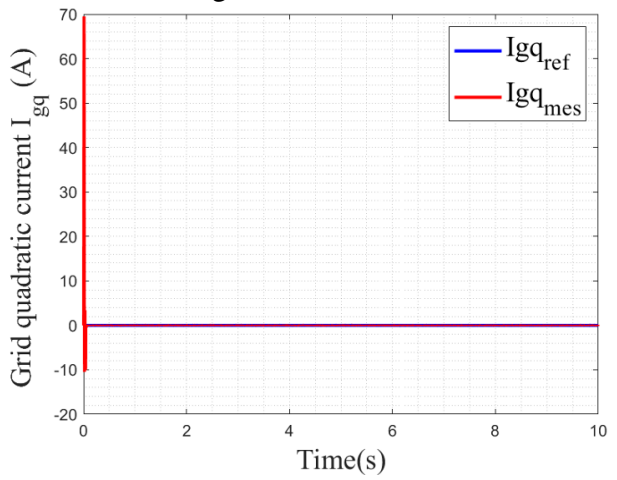

Figure 13. Gride quadratic current (reactive power)

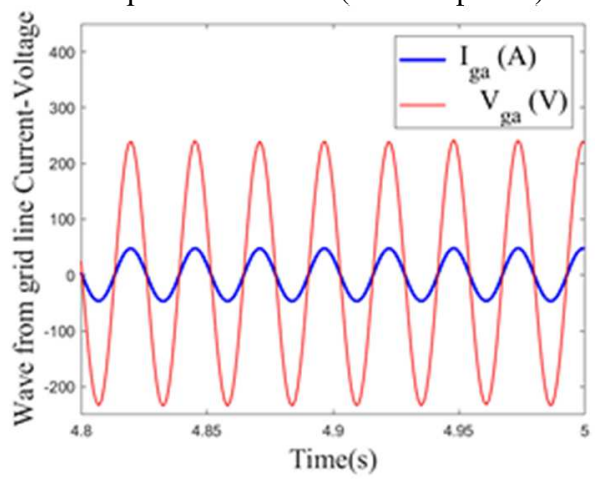

Figure 14. Wave frame of the line $i_{g a}$ current and $v_{g a}$ voltage 
Figure 8 is the mechanical rotor speed $\Omega$, perfectly converge to their respective references. Witch shows the robustness of the SMC command to track the estimated set point. In response to the chosen wind speed profile, the turbine generates the active power as shown in Figure 9. This active power corresponds to the optimal values of each wind speed.

Figures 10 and 11 show that the $d-q$ axis of the stator current $i_{\mathrm{sd}}$ and $i_{\mathrm{sq}}$, perfectly converge to their respective references. The tracking quality is quite satisfactory after each change in the wind speed, only there is a small difference between the setpoint and the measured signal in the transient state.

Figure 12 shows that the DC-link voltage $v_{D C}$ is perfectly regulated. The quadratic gride current $i_{q G}$ respectively the reactive power $Q_{g}$ injected into the grid is equals to zero, as shown in Figure 13 and Figure 14 shows the wave frame of the line current is all time sinusoidal and in phase with the grid voltage complying with the power factor correction requirement.

\section{Conclusion}

A SMC/Backstepping controllers for supported WECS ongrid has been proposed in this article. The control of the SSC has been assured by the proposed SMC includes NTSMC and SS-SMO in speed control loop and disturbance compensation loop in the field oriented controlled PMSG. The SMC not only controls the rotor speed but it is also responsible for reducing the chattering, mismatched disturbances and model uncertainties, through the disturbance compensation loop. A feed-forward term generated from SS-SMO, has been added to estimate the resultant q-axis stator current of the field oriented controlled PMSG. For the gride side inverter it's been controlled with an adaptive non-linear Backstepping voltage control technique to maintain the voltages at a preferred amplitude and frequency and to ensure the electrical grid currents must be sinusoidal with the frequency as the electrical voltages and the reactive power must be always zero. The control techniques have been verified through the simulation results. It has been observed that, the proposed technique is useful in smooth tracking of reference speed, reference q-axis stator current generation with reduced chattering and optimal wind power extraction. The results have confirmed the improved performance of the system under steady state and dynamic conditions.

\section{References}

[1] Sahu, A., Gupta, S., Singh, V. K., Bhoi, A. K., Garg, A., \& Sherpa, K. S. (2017). Design of Permanent Magnet Synchronous Generator for Wind Energy Conversion System. Lecture Notes in Electrical Engineering, 23-32. https://doi.org/10.1007/978-98110-4286-7_3

[2] Novaes Menezes, E. J., Araújo, A. M., \& Bouchonneau Da Silva, N. S. (2018). A review on wind turbine control and its associated methods. Journal of Cleaner Production, 174, 945-953. https://doi.org/10.1016/j.jclepro.2017.10.297
[3] Watil, A., El Magri, A., Raihani, A., Lajouad, R., Giri, F. Multi-objective output feedback control strategy for a variable speed wind energy conversion system (2020) International Journal of Electrical Power and Energy systems, 121, DOI: 10.1016/j.ijepes.2020.106081

[4] Watil, A. El Magri, A. Raihani, Nonlinear control of wind energy conversion system involving dg (2018) 43, doi:10.1145/3286606.3286820.

[5] El Magri, A., Giri, F., Abouloifa, A., Elfadili, A. Nonlinear control of a variable speed wind generator (2009) IFAC Proceedings Volumes (IFACPapersOnline), pp. 416-421.

[6] Ren, Y., Li, L., Brindley, J., \& Jiang, L. (2016). Nonlinear PI control for variable pitch wind turbine. Control Engineering Practice, 50, 84-94. https://doi.org/10.1016/j.conengprac.2016.02.004

[7] Pan, L., \& Shao, C. (2020). Wind energy conversion systems analysis of PMSG on offshore wind turbine using improved SMC and Extended State Observer. Renewable Energy, 161, 149-161. https://doi.org/10.1016/j.renene.2020.06.057

[8] Z. Jai Andaloussi, A. Raihani, A. El Magri, R. Lajouad, A. El Fadili, "Novel Nonlinear Control and Optimization Strategies for Hybrid Renewable Energy Conversion System", Modelling and Simulation in Engineering, vol. 2021, 20 pages, 2021. https://doi.org/10.1155/2021/3519490

[9] Do, T. D. (2017). Disturbance Observer-Based Fuzzy SMC of WECSs Without Wind Speed Measurement. IEEE Access, 5, 147-155.

[10]Douadi, T., Harbouche, Y., Abdessemed, R., Bakhti, I. (2018). Improvement Performances of Active and Reactive Power Control Applied to DFIG for Variable Speed Wind Turbine Using Sliding Mode Control and FOC. International Journal of Engineering, 31(10), 1689-1697.

[11]Lu, L. Y., Avila, N. F., Chu, C. C., \& Yeh, T. W. (2018). Model Reference Adaptive BackElectromotive-Force Estimators for Sensorless Control of Grid-Connected DFIGs. IEEE Transactions on Industry Applications, 54(2), 1701-1711.

[12]El Magri, A., Giri, F., Besançon, G., El Fadili, A., Dugard, L., Chaoui, F.Z. Sensorless adaptive output feedback control of wind energy systems with PMS generators, (2013) Control Engineering Practice, 21 (4), pp. 530-543. DOI: 10.1016/j.conengprac.2012.11.005

[13] Watil, A. E. Magri, A. Raihani, R. Lajouad, F. Giri, An adaptive nonlinear observer for sensorless wind energy conversion system with pmsg, Control Engineering Practice 98 (2020) 104356.

[14] Jamieson, P. (2018). Innovation in Wind Turbine Design (2nd ed.). Wiley.

[15] Reyes, V., Rodriguez, J. J., Carranza, O., \& Ortega, R. (2015). Review of mathematical models of both the power coefficient and the torque coefficient in wind turbines. 2015 IEEE 24th International Symposium on Industrial Electronics (ISIE). Published. https://doi.org/10.1109/isie.2015.7281688 
[16] Sitharthan, R., Karthikeyan, M., Sundar, D. S., \& Rajasekaran, S. (2020). Adaptive hybrid intelligent MPPT controller to approximate effectual wind speed and optimal rotor speed of variable speed wind turbine. ISA Transactions, 96, 479-489. https://doi.org/10.1016/j.isatra.2019.05.029

[17] Yin, M., Li, W., Chung, C. Y., Zhou, L., Chen, Z., \& Zou, Y. (2017). Optimal torque control based on effective tracking range for maximum power point tracking of wind turbines under varying wind conditions. IET Renewable Power Generation, 11(4), 501-510. https://doi.org/10.1049/iet-rpg.2016.0635

[18]El Magri, F. Giri, A. El Fadili, Control Models for Synchronous Machines,(2013) AC Electric Motors Control: Advanced Design Techniques and Applications, pp. 41-56. DOI: 10.1002/9781118574263.ch3

[19] Lajouad, R., El Magri, A., El Fadili, A., Chaoui, F.-Z., Giri, F. Adaptive nonlinear control of wind energy conversion system involving induction generator, (2015) Asian Journal of Control, 17 (4), pp. 13651376. DOI: $10.1002 /$ asjc. 1020

[20]Lajouad, R., Giri, F., Chaoui, F.Z., El Fadili, A., El Magri, A. Output feedback control of wind energy conversion system involving a doubly fed induction generator (2019) Asian Journal of Control, 21 (4), pp. 2027-2037, DOI: 10.1002/asjc.2116

[21]El Magri, A., Giri, F., Abouloifa, A., El Fadili, A. Nonlinear control of associations including wind turbine, PMSG and AC/DC/AC converters, (2014) 2009 European Control Conference, ECC 2009, pp. 4338-4343. DOI:10.23919/ecc.2009.7075082

[22] Errami, Y., Obbadi, A., Sahnoun, S., Ouassaid, M., \& Maaroufi, M. (2016). Proposal of a backstepping control strategy for dynamic performance improvement of PMSG Wind Farm with common DC bus. 2016 International Renewable and Sustainable Energy Conference (IRSEC). 